\title{
Dynamic Electron and X-ray Imaging is a Moving Experience
}

Simon Burgess ${ }^{1}$, Haithem Mansour ${ }^{1}$, Anthony Hyde ${ }^{2}$, Philippe Pinard ${ }^{3}$, Peter Statham ${ }^{3}$, Christian Lang ${ }^{4}$ and Michael Hjelmstad ${ }^{5}$

${ }^{1}$ Oxford Instruments NanoAnalysis, High Wycombe, England, United Kingdom, ${ }^{2}$ Oxford Instruments, England, United Kingdom, ${ }^{3}$ Oxford Instruments NanoAnalysis, High Wycombe, United Kingdom, ${ }^{4}$ Oxford Instruments Nanoanalysis, High Wycombe, England, United Kingdom, ${ }^{5}$ Oxford Instruments Inc, Pleasanton, California, United States

Live electron and X-ray imaging [1] or live chemical imaging [2] is a newly developed capability for sample investigation and navigation in electron microscopy. It uses simultaneous acquisition of electron images and EDS X-ray maps to provide morphological, chemical, and elemental images of a sample while it is being investigated in the SEM. The aim is to change the SEM workflow for sample investigation from a sequential to a parallel one. Traditionally, a field of view, FOV, of possible interest is identified using electron imaging then EDS mapping on this static FOV is used to check if this area is useful. In the new parallel workflow, useful regions for analysis can be identified from topographic and elemental information being presented while the FOV is changing. Some manufacturers have already improved the static workflow by presenting the electron image while moving the sample and automatically switching on X-ray acquisition when the FOV is stationary so that the display of elemental information builds up over many seconds or even minutes. Waiting until the FOV is stationary to obtain elemental information is still inefficient. Our aim has been to provide a more dynamic experience where augmenting the electron image with elemental information on the fly allows sites of interest to be identified far more effectively.

Dynamic electron and X-ray imaging requires a real time data refresh at frame rates of significantly less than 1 second. This allows an analyst to monitor changes in topography and element composition when changing microscope conditions or moving the sample to identify areas of interest. This 'Moving Experience' with sub-second feedback allows searching to be 10-100x faster than the "move-stop-acquirecheck" approach where only the electron image is available when moving. To achieve this goal, the response and refresh of X-ray elemental information needs to be as close as possible to the interactive experience a user would obtain from a secondary or backscatter detector. This is a challenge for EDS due to its inferior signal to noise $(\mathrm{S} / \mathrm{N})$ compared to electron imaging. X-ray count rates can be improved by working at high beam currents, but this compromises the electron image resolution and may be impossible with beam sensitive samples. Therefore, $\mathrm{X}$-ray $\mathrm{S} / \mathrm{N}$ is best improved by increasing the collection solid angle. To provide useful elemental information in real time requires accurate automatic identification of elements present, without false positives due to pile-up coincidence peaks due to the high count rates. A special technique was therefore developed to process this EDS data and cluster into sum spectra from areas of similar composition to allow accurate removal of pile-up artefacts and improve detection of elements concentrated into small regions of the field of view [1].

Since this technique was first introduced to SEM by Oxford Instruments in 2017 we have been developing the technique to improve image visualization, response, and interactivity. Improvements have been made in several areas. By using more or larger SDD sensors and moving them closer to the sample, the X-ray count rate is increased. The X-ray signal can be significantly enhanced with the same or even reduced beam current so that lower element concentrations can be detected in a changing FOV. With increased Xray signal and streamlining the processing and data handling of X-ray spectra, the frame rate of image 
display can be improved to enable a more dynamic experience. Frame rates are now fast enough to show a smooth evolution of a changing FOV without the distracting update of a slow raster progressing down the image. New techniques have also been developed to process and display the pixel intensities on the fly. This includes the display of individual X-ray maps, peak and overlap corrected maps ("TruMap"), and combined images where elemental information is overlaid on or mixed with electron images to give combined information in a single image. These developments make recognition of smaller features or more subtle element variations practical even at these higher frame rates. When the FOV is not changing, the data is accumulated rather than refreshed and with much higher X-ray count rates, feature details can be confirmed in seconds.

Dynamic electron and X-ray imaging provides a new paradigm for sample characterization in the SEM, making identification of features, structures and defects a more efficient and effective process by the addition of real-time elemental information. Elemental analysis no longer needs to be a stop/start, sequential process, with success driven by extensive sample knowledge, interpretation, extrapolation of electron image information by the user, and a degree of luck; it can now be a moving experience, helping users quickly achieve success with their exploration and analysis.

\section{References}

[1] P. Pinard et al. Microsc. Microanal. 24 S1 (2018), p. 624.

[2] A. Hyde et al. Microsc. Microanal. 25 S2 (2019), p, 1276 